\title{
A false case of infection caused by Dicrocoelium dendriticum
}

\author{
Cinzia Rossi', Claudia Canale', Franco Gandini', Leonardo Lodolo', Nino Cappuccia' \\ I Laboratorio Analisi Chimico Cliniche e Microbiologiche ASL VCO, Ospedale di Verbania \\ 2 Medico di Medicina Generale ASL VCO
}

Key Words: Anamnesis, diagnosis, Dicrocoelium dendriticum, stool parasitological exam

\section{Segnalazione di un caso di infestazione spuria da Dicrocoelium dendriticum}

\section{SUMMARY}

We describe a false case of infection caused by Dicrocoelium dendriticum, a cosmopolite trematode that can infect human bile ducts but tends to live in cattle or other grazing mammals. Our aim is to stress the relevance of adequate diagnostic methods and of exact medical history in order to detect any possible clinical case.

\section{INTRODUZIONE}

Dicrocoelium dendriticum è un trematode cosmopolita, presente anche in Italia, che può essere causa di gravi infezioni essenzialmente in ruminanti, come ovini e bovini, nei quali è tipicamente ospite definitivo. Il primo ospite intermedio di questo parassita sono molluschi di varie specie, mentre il secondo ospite intermedio è rappresentato dalle formiche $(2,4)$.

Il ciclo vitale inizia quando un mollusco ingerisce le uova con le feci eliminate da un animale infestato; qui le uova si sviluppano a sporocisti e quindi a cercarie; queste ultime vengono ingerite dalle formiche, nelle quali si sviluppano a metacercarie. L'ospite definitivo si infesta ingerendo le formiche parassitate che, per lesioni al loro sistema nervoso provocate dalle metacercarie, perdono la capacità di muoversi e restano bloccate sull'erba che l'erbivoro ingerisce $(2,3)$.

Data la specificità del ciclo vitale di questo trematode, le forme di parassitismo vero nell'uomo da Dicrocoelium dendriticum sono piuttosto rare e, quando reali, sono responsabili di un'infestazione dei dotti biliari, con conseguenti coliche biliari, disturbi generali nella digestione, inclusi gonfiore e diarrea (4).

\section{DESCRIZIONE DEL CASO}

G.C., donna di 43 anni, nata in Kazakistan, in Italia per lavoro e di professione badante, si presenta nel Novembre 2010 nel nostro Laboratorio per un esame coproparassitologico. La paziente consegna in Laboratorio il campione di feci corredata di scheda relativa alla ricerca dei parassiti fecali compilata in tutte le sue parti. Da tale scheda si evincono le seguenti informazioni: la signo- ra ha soggiornato per due mesi in Russia (da Aprile a Maggio 2010) e dichiara di presentare una sintomatologia aspecifica rappresentata da diarrea, perdita di peso e dolori addominali.

\section{Iter operativo - diagnostico}

Sul campione di feci pervenuto in Laboratorio vengono condotte osservazioni dapprima microscopiche dirette in soluzione fisiologica e in soluzione di Dobell, e quindi viene eseguito un arricchimento per concentrazione formolo-etilacetato (Para Pak Plus Ecofix, Meridian) (1,2).

All'osservazione microscopica dopo concentrazione si osserva un discreto numero di uova, di colorazione leggermente pleomorfa: sia bruno chiare che bruno scure.

Le dimensioni delle uova, opercolate, sono di circa 40-45 x 20-26 $\mu \mathrm{m}$ (Figura I) $(1,4)$. Ad una prima osservazione microscopica a piccolo ingrandimento $(10 \mathrm{x})$ si pensò alla possibilità di uova di Opisthorchis o simili, in virtù anche se non soprattutto della anamnesi fornita (la donna proveniva dal Kazakistan).

L'attenta osservazione delle caratteristiche (morfologia, colore e dimensioni reali) delle uova (40x) portano alla diagnosi di Dicrocoelium dendriticum. Riportiamo pertanto le nostre considerazioni ed il nostro percorso "conoscitivo" (2).

Tali uova sono da considerare uova spurie (di transito passivo) di Dicrocoelium dendriticum, un trematode comune anche in Italia, verosimilmente di nessun significato clinico in sé per sé.

Questo in quanto all'anamnesi iniziale non emergono elementi tali da giustificare un'infestazione vera e propria, e poi perché, come più avanti riferito, a successive indagini copro parassitologiche

\section{Corresponding author: Cinzia Rossi}

Laboratorio Analisi Chimico Cliniche e Microbiologiche ASL VCO

Via Crocetta,Verbania- Tel.: 0323 54I227; Fax: 0323 54I 247

E-mail: microbiologia@aslvco.it 
tali uova non verranno più reperite.

Le infestazioni (definite spurie o false infestazioni), con la successiva momentanea (pochi giorni) emissione di uova dette di transito passivo da Dicrocoelium dendriticum si verificano infatti in seguito a ingestione di alimenti animali, in particolare fegato, contenenti tali uova (quando la cottura non è stata adeguata).

Le infestazioni vere sostenute da tale parassita sono rare, se non eccezionali (trattabili peraltro sempre con Praziquantel o Albendazolo), in relazione soprattutto alle modalità di contaminazione ed infestazione, nell'uomo assai improbabile $(2,4)$.

Si decide così di contattare il medico curante per meglio comprendere il caso.

Viene riferito che a Luglio la paziente si era presentata dal medico curante lamentando disturbi intestinali e dimagrimento, ma gli esami ematologici per la ricerca di indici infiammatori e la coprocoltura risultavano negativi (fu condotta la ricerca colturale di Salmonella, Shigella e Campylobacter).

In Agosto venivano ripetute le analisi del sangue di routine che risultavano essere nella norma, così come gli ormoni tiroidei e le IgA anti - transglutaminasi.

A Ottobre alla paziente veniva prescritta una gastroscopia e a Novembre una pancolonscopia: entrambe le indagini non hanno rivelato alcuna anomalia funzionale o strutturale.

A fronte dell'esito dell'esame coproparassitologico positivo eseguito a Novembre e del fatto che la paziente è sintomatica (sia pure una sintomatologia del tutto aspecifica), il medico curante prescrive comunque una terapia con Albendazolo: $10 \mathrm{mg} / \mathrm{kg} /$ die due volte al di per 7 giorni.

Al termine della terapia la paziente riferisce al medico curante un miglioramento del suo stato di salute (effetto placebo?).

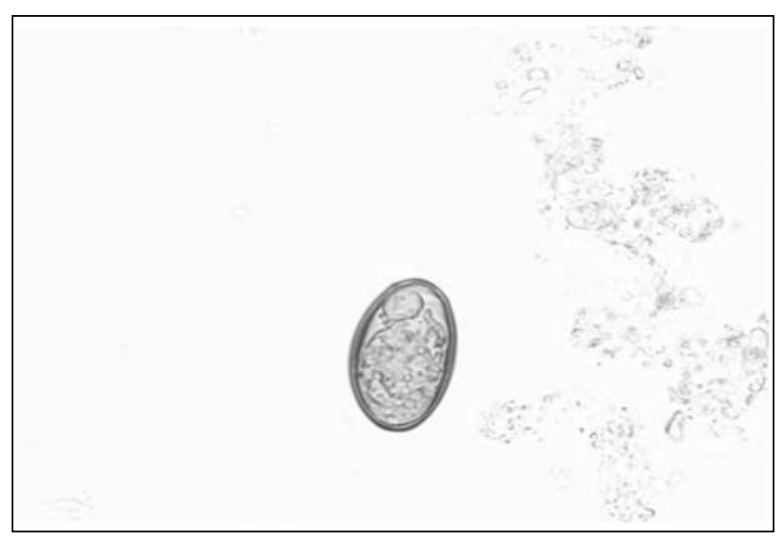

Figura I. Osservazione microscopica. Uovo di Dicrocoelium dendriticum in campione di feci dopo arricchimento per concentrazione formolo-etilacetato.
Il campione fecale analizzato successivamente per controllo ha dato esito negativo.

Dato che l'analisi microscopica delle uova ha portato alla "diagnosi" di una probabile infestazione spuria da Dicrocoelium dendriticum manca ancora il nesso causale con l'ingestione delle uova.

Si ricontatta pertanto nei mesi successivi e non senza qualche difficoltà la paziente, la quale riferisce di aver mangiato in Settembre, quindi quando era in Italia, del fegato di capra cucinato alla griglia.

Tale elemento, in armonia alle indagine copro parassitologiche, confermerebbe la possibilità se non la certezza dell'infestazione spuria.

\section{CONSIDERAZIONI CONCLUSIVE}

Segnaliamo questo presunto caso di infestazione spuria da Dicrocoelium dendriticum, che si può presentare più frequentemente di quanto si possa pensare, allo scopo di sottolineare l'importanza di percorsi diagnostici e anamnestico-clinici adeguati per comprendere adeguatamente ogni caso clinico si presenti alla nostra attenzione, vero o falso che esso sia.

\section{Ringraziamenti}

Si ringrazia il Dr. Daniele Crotti per la preziosa collaborazione alla diagnostica microscopica e alla descrizione del caso.

\section{BIBLIOGRAFIA}

1. Bernieri F, Crotti D, Galli D, Raglio A. Manuale illustrato di diagnostica parassitologia. Edizione Selecta Medica, Pavia, 2001.

2. D'Annibale ML. Infestazione spuria da Dicrocoelium dendriticum. Microb Med 2004; 19 (4): 399-400.

3. Duchacek L, Lamka J. Dicroceliosi-the Present State of Knowledge with respect to Wildlife Species. Acta Vet. BRNO 2003; 72: 613-26.

4. Pampiglione - Canestri Trotti. Dicrocoelium dendriticum In: Guida allo studio della Parassitologia, Società Editrice Esculapio, Bologna, III Edizione, 1999; 145-7.

5. Petithory JC, Ardoin-Guidon F. Vrais et faux parasites en coprologie microscopique, Cahier de formation, BIOFORMA, n.3, 1995. 